\title{
ICU Continuous Monitoring Data Analysis Based on Online Segmentation Algorithm
}

\author{
Yongfei Chen ${ }^{1}$, Bugao Zhou ${ }^{2 *}$ \\ ${ }^{1}$ College of Computer, Jiangxi University of Traditional Chinese Medicine, Nanchang, 330004, \\ China \\ ${ }^{2}$ College of Science and Technology, Jiangxi University of Traditional Chinese Medicine, \\ Nanchang, 330004, China \\ bugao_zhou@yeah.net \\ *Corresponding author
}

Keywords: Segmentation algorithm, Intensive care, Continuous monitoring, Ant colony distribution, VB software, Cloud.

\begin{abstract}
In order to improve the comfort of ill patients admitted during the ICU, reduce the pain caused by various operations, improve the efficiency of data monitoring in ICU and analyze the sample data, this paper puts forward a kind of continuous data analysis algorithm based on online segmentation. Through the statistics of 3000 patients pain data samples in ICU, this paper obtains the ant colony distribution of pain level data, and uses VB software to segment ant data with different coefficients, finally gets the pain level 3D cloud for different levels, which provides a theoretical basis for the study on electronic equipment used in ICU.
\end{abstract}

\section{Introduction}

Most critically ill patients have experienced bad memory in the ICU, and pain caused by operation is one of the main bad memories. Some conventional nursing measures such as removal of drainage tube, wound care, deep breathing, cough practice, sputum suction operation, changing position will give ill patients pain. Not control of pain may lead to multiple organ complications, duration of chronic pain, anxiety and other adverse consequences [1]. But most critically ill patients have consciousness disorder and tracheal intubation factors, they can't express the pain. In order to improve the efficiency of the patient's nursing, it is effective to detect and continuous evaluate the ward electronic equipment data. Based on this, this paper designs the data monitoring algorithm of ICU, and the main framework is as follows:

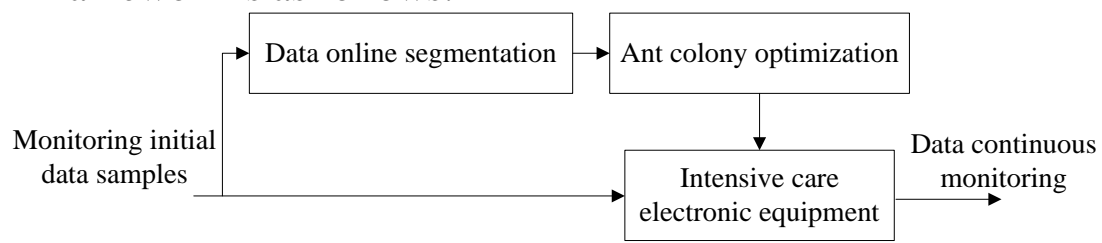

Fig.1: The data sample online segmentation process

As shown in Figure 1 it indicates the basic frame chart of data sample online segmentation. Initial sample data is firstly through genetic binary code, using equidistant partition coefficient to rank sample, and using the ant colony algorithm to optimize data, eventually realizing the data continuous monitoring [2]. This monitoring value has an important guiding significance for the ICU electronic equipment operation.

\section{Data Online Segmentation Algorithm Theoretical Basis}

We use particle filter theory to simulation model of the relationship between the levels[3]. Specifically, our goal is based on the observation and background of the regional estimation of the posterior density[4]. According to Bias's rule, we can get update rules of the dynamic graphical models . 


$$
\begin{gathered}
p\left(x_{1: t} \mid x_{1: t}^{\prime}, z_{1: t}, z_{1: t}^{\prime}\right) \\
=\frac{p\left(x_{t}, x_{t}^{\prime}, z_{t,} z_{t}^{\prime} \mid x_{1: t-1}, x_{1: t-1}, z_{1: t-1}, z_{1: t-1}\right)}{p\left(x_{t}^{\prime}, z_{t}, z_{t}^{\prime} \mid x_{1: t-1}^{\prime}, z_{1: t-1}, z_{1: t-1}^{\prime}\right)} p\left(x_{1: t-1} \mid x_{1: t-1}^{\prime}, z_{1: t-1}, z_{1: t-1}^{\prime}\right) \\
\chi=\frac{p\left(z_{t}, z_{t}^{\prime} \mid x_{t}, x_{t}^{\prime}, x_{1: t-1}, x_{1: t-1}^{\prime}, z_{1: t-1}, z_{1: t-1}^{\prime}\right)}{p\left(x_{t}^{\prime}, z_{t}, z_{t}^{\prime} \mid x_{1: t-1}^{\prime}, z_{1: t-1}, z_{1: t-1}^{\prime}\right)} p\left(x_{t}, x_{t}^{\prime} \mid x_{1: t-1}, x_{1: t-1}^{\prime}, z_{1: t-1}, z_{1: t-1}^{\prime}\right) \\
=\frac{p\left(z_{t}, z_{t}^{\prime} \mid x_{t}, x_{t}^{\prime}\right) p\left(x_{t}, x_{t}^{\prime} \mid x_{1: t-1}\right)}{p\left(x_{t}^{\prime}, z_{t}, z_{t}^{\prime} \mid x_{1: t-1}^{\prime}, z_{1: t-1}, z_{1 ! t-1}^{\prime}\right)} p\left(x_{1: t-1} \mid x_{1: t-1}^{\prime}, z_{1: t-1}, z_{1: t-1}^{\prime}\right) \\
=\frac{p\left(z_{t} \mid x_{t}\right) p\left(z_{t}^{\prime} \mid x_{t}^{\prime}\right) p\left(x_{t}^{\prime} \mid x_{t}\right) p\left(x_{t} \mid x_{t-1}\right)}{p\left(x_{t}^{\prime}, z_{t}, z_{t}^{\prime} \mid x_{1: t-1}^{\prime}, z_{1: t-1}, z_{1: t-1}^{\prime}\right)} p\left(x_{1: t-1} \mid x_{1: t-1}^{\prime}, z_{1: t-1}, z_{1: t-1}^{\prime}\right)
\end{gathered}
$$

In the formula (2), we use the conditions independent attributes of the graphical model, in the formula (4), we apply these characteristics, based on the theory of particle filtering, and posterior density can be expressed as:

$$
p\left(x_{1: t} \mid x_{1: t}^{\prime}, z_{1: t}, z_{1: t}^{\prime}\right) \approx \sum_{n=}^{N} w_{t}^{n} \delta\left(x_{1: t}-x_{1: t}^{n}\right)
$$

In formula (5) $\left\{x_{1: t}^{n}, n=1,2, \ldots, N\right\}$ is a sample set, $\left\{w_{t}^{n}, n=1,2, \ldots, N\right\}$ is the associated weighting, $\delta$ is the Dirac function. Weight can be given based on the importance of sampling theory:

$$
w_{t}^{n} \infty p\left(z_{t} \mid x_{t}\right) p\left(z_{t}^{\prime} \mid x_{t}^{\prime}\right) p\left(x_{t}^{\prime} \mid x_{t}\right) w_{t-1}^{n}
$$

In the formula (6), $p\left(z_{t} \mid x_{t}\right)$ and $p\left(z_{t}^{\prime} \mid x_{t}^{\prime}\right)$ are the possibility of the target observed and the background observed, they can be estimated by the image observation data. In our experiments, we used a weight based on the histogram of the nuclear theory to give a sample. In particular, we can estimate the local likelihood density as follows:

$$
p\left(z_{t} \mid x_{t}^{n}\right)=\frac{1}{2 \pi \sigma_{1}} \exp \left\{-\frac{\left\{h\left(z_{t} \mid x_{t}^{n}\right)-h_{0}\right\}^{2}}{\sigma_{1}^{2}}\right\}
$$

$h_{0}$ in the type is the initial histogram model of the target object, $h\left(z_{t} \mid x_{t}^{n}\right)$ is a histogram of the samples x. Similarly, we have the likelihoods density of background:

$$
p\left(z_{t}^{\prime} \mid x_{t}^{m}\right)=\frac{1}{2 \pi \sigma_{2}} \exp \left\{-\frac{\left\{h^{\prime}\left(z_{t} \mid x_{t}^{n}\right)-h_{0}^{\prime}\right\}^{2}}{\sigma_{2}^{2}}\right\}
$$

$h_{0}$ is the initial histogram model of the target object, $h^{\prime}\left(z_{t} \mid x_{t}^{n}\right)$ is called the "object - the relationship between background density”. Because we use a fixed width to select the background with a predefined, in our experiments, we assume that this is a Gaussian distribution. When a posteriori estimation iteration, it can be calculated the expectancy to give the final estimated shape of the object.

Because in an iterative case, it is very difficult to estimate of object contour according to one step, we propose a dynamic graphical model, $t$ is an iteration index respectively, ${ }^{z_{t}}$ and ${ }^{z_{t}}$ said image observation space target and background regions of pixel set. The intermediate layer is based on graph theory [5].It can be thought the directly relationship has the same direction from PCA shape space to the pixel state space of a direct link with the pixels from state space to image observation space. 

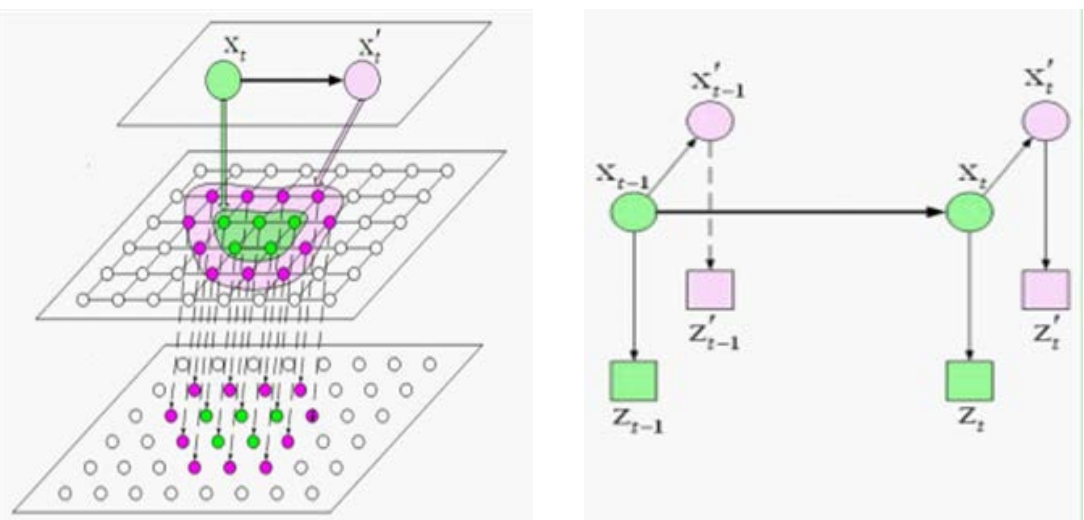

Fig.2: System graphical model

Data online segmentation is based on online distribution statistics of data samples, and divides the different data samples based on grades, and encodes the data using different encoding forms [6]. The general use optimum coding is the binary genetic algorithm, and the model is as shown in the formula (9).

$$
\left.A=\min +\sum_{i=1}^{m} a_{i} 2^{i-1}\right) \frac{\max -\min }{2^{m}-1} .
$$

This paper uses limit value to process the two similar data samples and optimization. The relationship of data fusion is as follows:

$$
C(x, y)=\iint(M-R) d x d y
$$

When the function values of $C$ is infinitesimal, data sample $M$ will be close to data samples $N$ infinite. Desecrating the sample data:

$$
C(x, y)=\sum_{b=1}^{m} \sum_{a=1}^{n}(M-R)^{2}
$$

Assume that the data samples $M$ and $N$ have overlap, overlapping parts of them respectively are $M_{1}(x, y), N_{1}(x, y), p$ is the gain coefficient from $M_{1}$ to $N_{1}, q$ is zero line offset coefficient from $M_{1}$ to $N_{1}[7,8]$. When the values obtained are the smallest, it can do segmentation optimization on sample data.

$$
D(x, y)=\iint(p M+q-R) d x d y .
$$

The formula of data samples online segmentation through discretion is as follows:

$$
D(a, b)=\sum_{b 0=1}^{m} \sum_{a 0=1}^{n}(p M 0+q-R 0)^{2}
$$

The entire sample data can use particle swarm optimization. The optimal position of particle swarm search is the global optima, denoted as:

$$
g_{\text {best }}=\left(p_{g 1}, p_{g 2}, \cdots, p_{g D}\right) \text {. }
$$

Finding the two optimal values, by updating the data particle re-evaluates the data online segmentation effect, and divides the data again, realizing iterative optimization process of data segmentation.

$$
\begin{aligned}
& v_{i d}=w * v_{i d}+c_{1} r_{1}\left(p_{i d}-x_{i d}\right)+c_{2} r_{2}\left(p_{g d}-x_{i d}\right) \\
& x_{i d}=x_{i d}+v_{i d}
\end{aligned}
$$

In order to realize the data online segmentation, this paper uses VB programming way to program algorithm, and the main algorithms used are as follows:

for $\mathrm{i}=1: \mathrm{N}$

$$
\text { for } j=1: D
$$




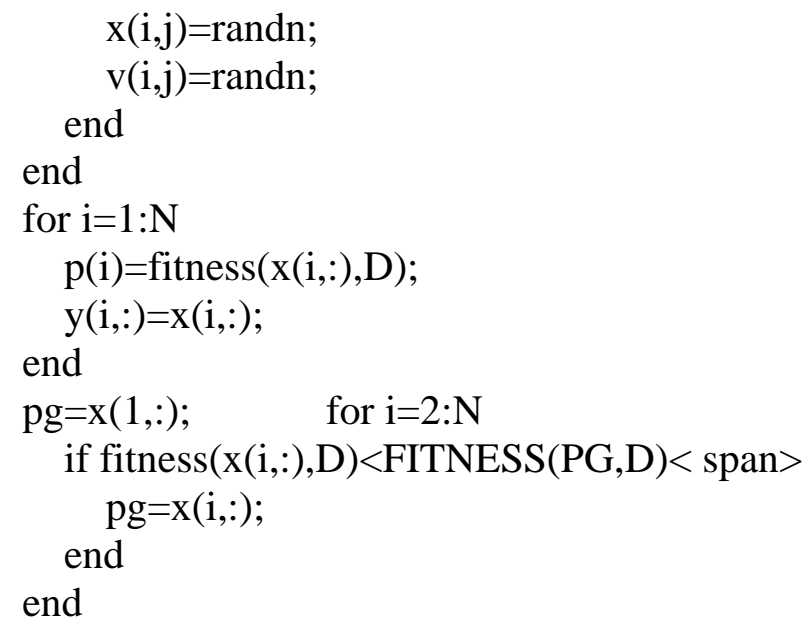

\section{Research on ICU Electronic Equipment Monitoring Data}

In order to verify the effectiveness and reliability of the data online segmentation algorithm designed in section second, this paper uses VB data processing toolbox to do online segmentation on ICU large data. The schematic diagram of equipment in ICU is as shown in Figure 3.

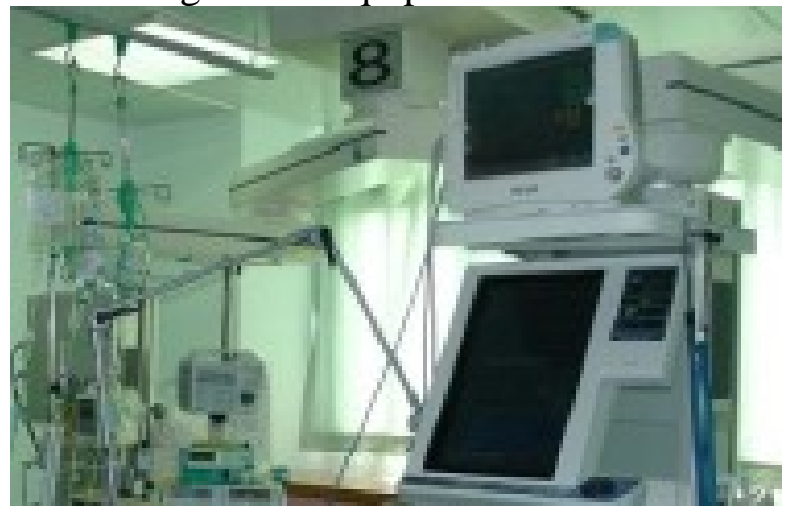

Fig.3: ICU internal electronics

Figure 3 shows the diagram of electronic equipment within the ICU. Large amount of calculation for the ICU device is not a problem, as shown in Figure 3; the computing device in ICU doesn't have too much requirement on the volume, so they usually have big storage capacity and the large amount of calculation[9].

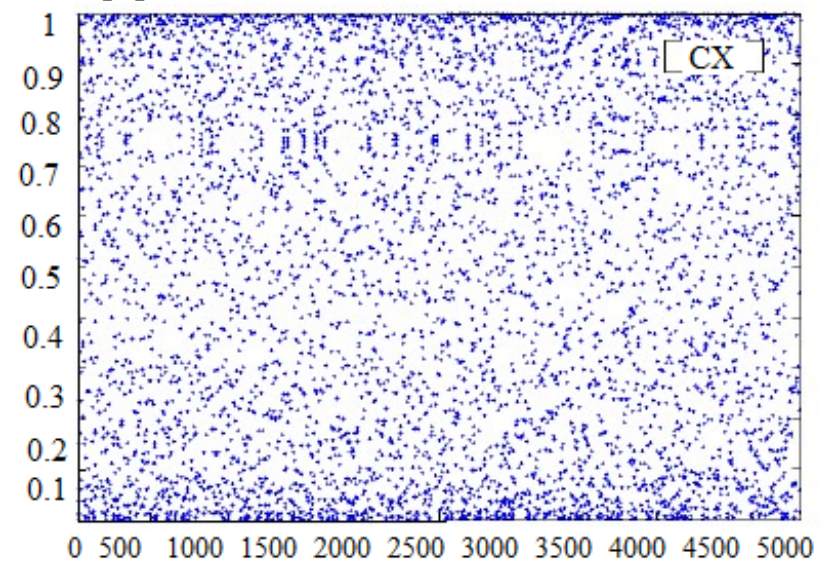

Fig.4: Pain data statistics for intensive care patients

Figure 4 represents data statistical sample. Through the analysis of 3000 intensive care patients' data and different levels division of pain behavior, this paper obtained the distribution map of the patient's pain[10]. And it uses the online segmentation to segment the data, the result is as shown in Figure 5. 


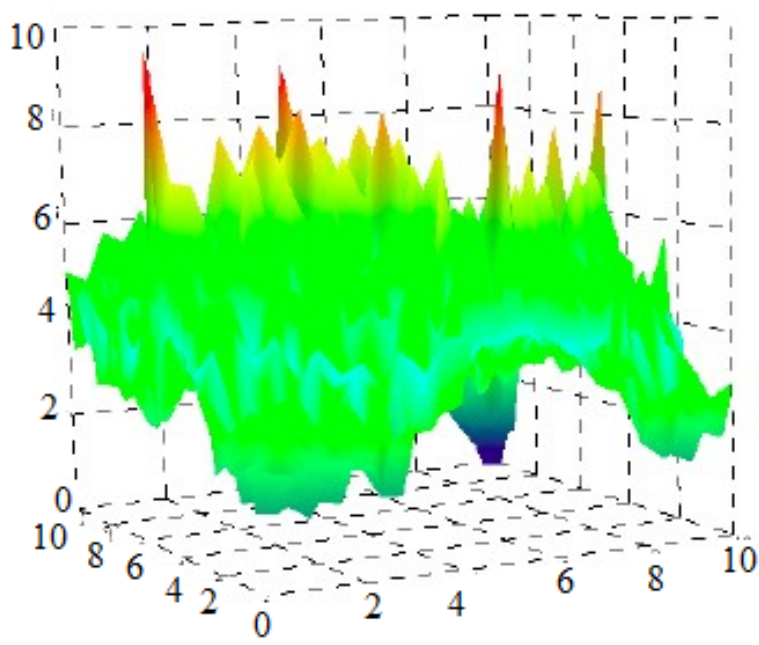

Fig.5: 3D online segmentation for monitoring data

Figure 5 shows the online segmentation results for 3D data. It divides the intensive care patient's pain into ten levels, and uses different segmentation interval to do online segmentation on the data, the results obtained are as shown in Figure 4 . Red is higher pain behavior level, it should be emphatically considered in nursing[11,12].

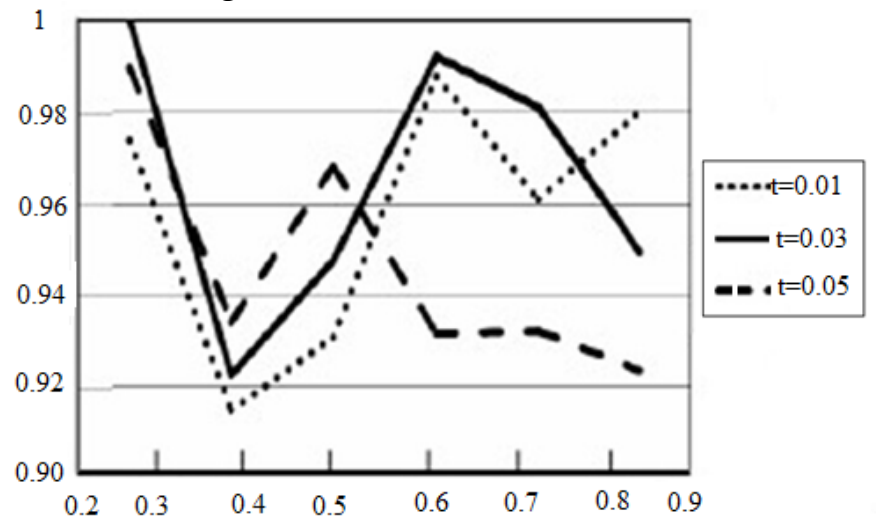

Fig.6: The accuracy rate with different partition coefficients

Figure 6 shows the data monitoring interval accuracy rate under different segmentation interval. Through the detecting of 0.2-0.9 seconds, the accuracy rate has obvious fluctuation, but when the interval is 0.01 and 0.03 , the accuracy is better than that in 0.05 . So in the online data segmentation, it needs to consider the segmentation interval to improve the accuracy of intensive care data detecting. The accuracy of ICU data can affect the analysis of the pathological severity and effectively improve the efficiency of the patient nursing.

\section{Summary}

This paper designs the continuous monitoring method of ICU electronic equipment large data, and uses the binary genetic algorithm to encode the data distribution, use equidistant to do online segmentation on the data samples, finally the data monitoring program under the VB software operating environment is obtained. Through the statistical and numerical calculation for 3000 ICU patients pain data, the pain level 3D distribution is obtained, which provides reference for nursing care for critical patients. For the other data not pain data processing, it needs to be further studied. Through the use of different data processing algorithms, it can make the data online segmentation more accurate.

\section{References}

[1] X.X. Fu, Y.D. Zhang, J. Guo. Explore and implement of ICU nursing performance management. China practical medicine, 2013, 2(12): 45-49. 
[2] C.S. Yang, X.H. Qiu, Y.Z. Huang, J,Y. Xu, Y. Yang, N.Y. Sun, D.D. Dong, H.B. Qiu. Status analysis and suggestion of classification management for ICU patient. China health quality management, 2013, 4(1): 23-25.

[3] L.P. Gu, L.H. Qiu, Y.M. Sun. Establishment and implementation of the ICU nursing performance appraisal system. Shanghai nursing, 2012, 5(3): 36-39.

[4] D.F. Cai, Y.C. Dong, C.Q. Li, Y. Zhou. Nursing performance assessment based on nurse in charge of the patient. Journal of nursing, 2012, 4(1): 34-35.

[5] X.P. Liu, H. Xie, F.P. Tao. Intensive care wards hierarchical monitoring progress. Journal of nursing, 2012, 5(21): 67-69.

[6] Z.Q. Chen, S.F. Zhang, S.F. Hu, M.J. Shen, W.H. An. Infection distribution and drug resistance analysis of pathogenic bacteria infection. Chinese journal of hospital infection, 2013, 5(7): 101104.

[7] J.B. Ma, S.J. Li, D.L. Cui, Z.G. Wu, B.Z. Ling, L.H. Pan. The distributions and changes of bacterial infection and drug resistance of pathogens in 2005-2009 years. Chinese journal of hospital infection, 2012, 4(6): 23-28.

[8] Q. Zhong, C.H. Wang, X.L. Gao, C. Duan, H. Li. Prevention and control of neonatal nosocomial transmission of multi drug resistant bacterial infection. Chinese Journal of hospital infection, 2013, 5(7): 112-115.

[9] L.Y. Cui, J. Lu, W.T. Luo, J.Y. Zhao, X.Y. Song, W.B. Liu. 2007-2009 years of Acinetobacter baumannii Bacillus drug resistance analysis. Chinese Journal of hospital infection, 2013, 1(2): 11-15.

[10] G.Q. Chen, H.Y. Cao, Z.G. Yao, J. Li, R.F. Gui. ICU Acinetobacter baumannii resistance survey. Chinese Journal of hospital infection, 2012, 3(4): 21-23.

[11] N. Zhou, C.Q. Luo, L. Guo. Distribution of pathogenic bacteria infection in ICU and drug resistance characteristics. Chinese Journal of hospital infection, 2013, 2(4): 47-49.

[12] H.B. Lei, W. Fan, E. Huang, G.M. Yi, L.S. Duan. Investigation analysis of respiratory tract infection of gram negative bacilli drug resistance in ICU. Chinese Journal of hospital infection, 2012, 3(1): 78-80. 